\title{
CONDUCTAS DE RIESGO AUDITIVO Y ACCIÓN DEL MECANISMO DE PROTECCIÓN COCLEAR EN ADOLESCENTES*
}

\section{AUDITORY RISK BEHAVIORS AND THE ACTION OF COCHLEAR PROTECTION MECHANISM IN ADOLESCENTS}

\author{
María Hinalaf ${ }^{* *}$, E. Cristina Biassoni*** Mónica Abraham ${ }^{* * * *}$ \\ JoRge Pérez VILLALOBO ${ }^{* * * * *}$, ANA L. MAGGI ${ }^{* * * * * *}$, SILVIA JOEKES S $^{* * * * * * *}$ Y \\ MeRCEDES X. HÜG*
}

*Investigación financiada con becas de formación de postgrado asignadas a la primera autora, de la Secretaria de Extensión Universitaria de la Universidad Nacional de Córdoba (UNC) y del Consejo Nacional de Investigaciones Científicas y Técnicas (CONICET), y con los subsidios Investigación multidisciplinaria dirigida a la Conservación y Promoción de la Audición en adolescentes de Escuelas Técnicas de la Ciudad de Córdoba. Fase II del CONICET y Promoción y prevención de la Audición en adolescentes de Escuelas Técnicas y su relación con hábitos recreativos Fase II otorgado por la Facultad Regional Córdoba de la Universidad Tecnológica Nacional (UTN), ambos dirigidos por la Lic. E. Cristina Biassoni y codirigidos por el Ing. Mario Serra.

${ }^{* *}$ Doctora en Ciencias de la Salud y Licenciada en Fonoaudiología. Becaria Post-doctoral del Consejo Nacional de Investigaciones Científicas y Técnicas (CONICET) en el Centro de Investigación y Transferencia en Acústica (CINTRA) y Docente de la Escuela de Fonoaudiología en la Facultad de Ciencias Médicas de la Universidad Nacional de Córdoba

(UNC). E-Mail: mariahinalaf@gmail.com

***Licenciada en Psicología y Especialista en Psicoacústica, Psicología Ambiental y Conservación de la Audición. Miembro de la Carrera del Investigador Científico del Consejo Nacional de Investigaciones Científicas y Técnicas (CONICET) y Codirectora de la Línea de Conservación de la Audición del Centro de Investigación y Transferencia en Acústica (CINTRA). E-Mail: cbiassoni@gmail.com

${ }^{* * * *}$ Magister en Psicología, Educación y Desarrollo y Licenciada y Doctoranda en Psicología. Miembro del Centro de Investigación y Transferencia en Acústica (CINTRA) y Coordinadora del Proyecto de Investigación en Educación en

la Pontificia Universidad Católica de Chile y Asesora en Gestión Educativa. E-Mail: moniabraham5@gmail.com *****Ingeniero Electrónico, Especialista en Higiene y Seguridad en el Trabajo y Maestrando en Ingeniería Ambiental.

Miembro del Centro de Investigación y Transferencia en Acústica (CINTRA). Docente de grado y posgrado en la Facultad Regional Córdoba de la Universidad Tecnológica Nacional (UTN). E-Mail: jorgeperezvillalobo@gmail.com ******Licenciada en Fonoaudiología. Docente en la Escuela de Fonoaudiología de la Facultad de Ciencias Médicas de la Universidad Nacional de Córdoba (UNC) y Pasante en el Centro de Investigación y Transferencia en Acústica

(CINTRA). E-Mail: analuzmaggi@gmail.com

*******Licenciada en Estadística, Magister en Estadística Aplicada y Doctora en Ingeniería (Mención Control Estadístico de Calidad). Profesora Titular y miembro del Instituto de Estadísticas y Demografía (IED) de la Facultad de Ciencias

Económicas de la Universidad Nacional de Córdoba (UNC). E-Mail: joekess@eco.unc.edu.ar

********Doctora en Psicología. Miembro de la Carrera del Investigador Científico del Consejo Nacional de

Investigaciones Científicas y Técnicas (CONICET) y Docente de la Facultad de Psicología de la Universidad Nacional de Córdoba (UNC). E-Mail: mhug@scdt.frc.utn.edu.ar

Los autores agradecen la colaborción recibida a las autoridades, personal y alumnos de las instituciones escolares participantes y al Ministerio de Educación de la Provincia de Córdoba de Argentina.

Consejo Nacional de Investigaciones Científicas y Técnicas (CONICET) y

Centro de Investigación y Transferencia en Acústica (CINTRA). Universidad Tecnológica Nacional (UTN),

Facultad Regional Córdoba. Unidad Asociada al CONICET.

Mtro. M. López esq. Cruz Roja Argentina s/n (X5016ZAA) Ciudad Universitaria. Córdoba, República Argentina. 


\section{Resumen}

La exposición a ruido no ocupacional y a la música a alto nivel sonoro, constituye un creciente factor de riesgo de pérdida auditiva en adolescentes. Estudios internacionales postulan que una función importante del Sistema Eferente Medial (SEM) es proteger el oído interno frente al ruido mediante un mecanismo reflejo y que puede ser evaluado a través de la Supresión Contralateral (SC) de las otoemisiones acústicas transitorias (TEOAEs). El mecanismo subyacente de este fenómeno no se conoce en su totalidad y los antecedentes sobre el tema son escasos. En esta investigación se analizó la relación entre la $E x$ posición General a Música (EGM), el estado de la función auditiva y el mecanismo de protección coclear. Participaron 91 adolescentes con edades entre 14 y 15 años. Se evaluaron dos dimensiones. (a) Psicosocial: se examinó la EGM con un cuestionario de actividades extraescolares y (b) auditiva o sea el funcionamiento del SEM mediante $\mathrm{SC}$ de las TEOAEs y los perfiles audiométricos con audiometrías. Los resultados mostraron que la SC no influyó estadísticamente en los perfiles audiométricos y categorías de exposición. Sin embargo, los resultados de la SC se orientan hacia la menor magnitud de efecto supresor, disminución en la respuesta total en relación al descenso de los umbrales auditivos y en relación con las categorías alta y baja de EGM. Es necesario promover trabajos destinados al comportamiento de riesgo auditivo conjuntamente con el mecanismo protector del SEM. De esta manera se podrá contribuir en la identificación temprana de la sensibilidad auditiva en adolescentes expuestos a ruido no ocupacional.

Palabras clave: Exposición general a música; Comportamiento de riesgo auditivo; Sistema eferente medial; Mecanismo de protección coclear; Supresión contralateral de las otoemisiones acústicas; Adolescentes.

\section{ABSTRACT}

Non-occupational exposure to noise, such as loud music sound level is an increasing risk factor for hearing loss in adolescents. International stud- ies propose that an important function of the medial efferent system (MES) is protect the inner ear against noise by a reflex mechanism and can be evaluated through Contralateral Suppression (CS) of transient otoacoustic emissions (TEOAEs) to analyzing the presence or absence of suppressive effect. The absence of suppressive effect is considered a possible alteration of the MES and the role it exerts on the regulation of the outer hair cells. The underlying mechanism of this phenomenon is not known totality and the background about this topic are limited. However, researches suggests that MES would have an important role in protection against acoustic overstimulation under the hypothesis that a hearing with the presence of suppressive effect would tend to be more protected against exposure to high noise levels while ears with no effect suppressor may be more vulnerable to noise exposure. In this research the relationship between the General Exposure to Music (MGE), the state of hearing function and cochlear protection mechanism were analyzed in 91 adolescents with 14/15 years old, with male predominance, of two technical schools of the city of Córdoba (Argentina). Were evaluated the following dimensions: (a) Psychosocial: MGE through an Out-of-School Activities Questionnaire to know in detail the participation in five recreational activities to finally analyze the MGE and (b) Audiological: assessment of audiometric profiles, as a subjective method, by audiometry in the conventional frequency ranges (250-8000) $\mathrm{Hz}$ and an extended high frequency audiometry (8000-16000) Hz; function of MES assessment, objective method, through CS of TEOAEs to determine the presence or absence of suppressive effect. The study CS of TEOAEs consisted of two steps: Step 1, application of TEOAEs of an ear in form ipsilateral without acoustic stimulation in the contralateral ear (CAS) and Step 2, application of TEOAEs in the ear ipsilateral while applying CAS. Was considered presence of suppressive effect when there was a reduction in the response after the CAS that is to say when the difference of values obtained in the total response with and without CAS was positive. At the same time, was considered absence of suppressive effect in cases of maintained or increased the response after the CAS, obtaining a value of zero or negative. The results of the research showed that in the group with presence of suppressive effect the most of ears is 
among moderate and high categories of MGE with a lower average magnitude of suppressive effect and a decreased amplitude of the total response in the high category of MGE than the media category. Regarding audiometry the ears with presence of suppressive effect showed higher magnitude in the group of normal hearing thresholds compared with the group of descended hearing thresholds. The results related to CS of the TEOAEs statistically did not influence in the audiometric profiles and the exposure categories. However the results of the CS were oriented towards the smaller magnitude of suppressive effect, decreased total response related with the descended hearing thresholds and high and low categories of MGE. It is necessary to promote and intensify researches in relation to the auditory risk behaviors together with the protection mechanism of MES. In this way, more researches can contribute to the early identification of hearing sensitivity in adolescents exposed to non-occupational noise. It is important to implement a PsychoAuditory Screening to collaborate in the prevention and promotion of hearing health.

Key words: Musical general exposure; Auditory risk behaviors; Medial efferent system; Cochlear protection mechanism; Contralateral suppression of the transient otoacoustic emissions; Adolescents.

\section{INTRODUCCIÓN}

La hipoacusia inducida por ruido constituye en la actualidad un problema social y de la salud pública. La mayor parte de los esfuerzos han estado orientados a reducir los riesgos que provoca la exposición a ruido en los adultos y en los ambientes laborales, descuidando los estadíos previos del desarrollo. Sin embargo, las investigaciones previas informan sobre la tendencia cada vez más acentuada a sufrir pérdidas auditivas en niños y adolescentes (Blair, Hardegree \& Benson; 1996; Chung, Des Roches, Meunier \& Eavey, 2005; Niskar et al., 2001). Estudios realizados en el Reino Unido (Sadhra, Jackson, Ryder \& Brown, 2002; West \& Evans, 1990), Francia (Meyer-Bisch, 1996) y China (Morioka et al.,
1996) han mostrado evidencia del deterioro auditivo inducido por ruido en adultos jóvenes relacionado con actividades recreativas y de ocio.

Especialmente los adolescentes se exponen con frecuencia a lo que técnicamente se denomina ruido no ocupacional o también llamado ruido social. Ambos términos se refieren a todas las fuentes sonoras presentes en actividades de ocio, no relacionadas con el ambiente laboral (Biassoni, Serra, Pérez Villalobo, Joekes \& Yacci, 2008; Jofre et al., 2009; Zenker, Altahona \& Barajas, 2001).

Las fuentes de exposición a ruido no ocupacional o social son de variada etiología. En el caso de los adolescentes, las más importantes provienen de escuchar música a altos niveles sonoros, ya sea en el hogar, fuera de él o a través del uso de reproductores personales de música (Müller, Dietrich \& Janssen, 2010; Serra, M.R., Biassoni, Ortiz Skarp, Serra, M. \& Joekes, 2007; Serra et al., 1998). En este sentido, la exposición a música a altos niveles sonoros es considerada como un comportamiento de riesgo para la salud auditiva, teniendo en cuenta la frecuencia de exposición, la duración de la misma y los niveles sonoros (Biassoni et al., 2011; Bohlin \& Erlandsson, 2007; Hinalaf et al., 2011a; Hinalaf et al., 2011b; Hinalaf et al., 2011c; Serra et al., 2007; Widén, 2006; Widén \& Erlandsson, 2007).

A nivel internacional, existe una acentuada preocupación por el incremento de las pérdidas auditivas inducidas por ruido en edades cada vez más tempranas y a su vez por los efectos a largo plazo de la exposición a ruido no ocupacional en esas edades (Folmer, Griest \& Martin, 2002; National Institutes of Health [NIH], 2000; Wallhagen, Strawbridge, Cohen \& Kaplan, 1997). Por su parte, la Organización Mundial de la Salud (OMS, 1999) destaca la importancia de la detección temprana de este tipo de hipoacusias y la necesidad de implementar estrategias tendientes a su prevención.

En el contexto nacional, el Centro de Investigación y Transferencia en Acústica (CINTRA) con gran interés en el tema, desarrolla investigaciones multidisciplinarias con estudios longitudinales a fin de conocer a través del 
tiempo la relación de las variables audiológicas, psicosociales y acústicas involucradas en el comportamiento que caracteriza a los adolescentes y jóvenes de nuestros días con respecto a la exposición a música a altos niveles sonoros. Los resultados de uno de esos estudios mostraron en las siguientes dimensiones: (a) en la audiológica, descensos en los umbrales auditivos de todos los adolescentes evaluados en el cuarto año de estudio, más acentuados en algunos casos que en otros (Abraham et al., 2011; Biassoni et al., 2011), (b) en la psicosocial, aumento progresivo con la edad en la participación de actividades recreativas caracterizadas por altos niveles sonoros y (c) en la acústica, las mediciones de niveles sonoros en lugares bailables registraron valores entre (108 y $112 \mathrm{dBA}$ ) y durante el uso de reproductores personales de música entre $(83 \mathrm{y}$ $105 \mathrm{dBA}$ ). Los valores medidos superan los recomendados por la normativa nacional (Ley Nacional No 79587, 1972 y Resolución N ${ }^{\circ}$ 295 / 2003) e internacional ISO (2010) en lo que respecta a la exposición a ruido ocupacional, es decir, en los ambientes laborales (Serra, M.R., Biassoni, Ortiz Skarp, Serra, M. \& Joekes, 2007).

Estudios recientes muestran que la exposición continuada tanto a ruido ocupacional como a recreativo, en edades tempranas, podría ocasionar desplazamientos permanentes del umbral auditivo (daño auditivo irreversible) a lo largo del tiempo debido a la acumulación gradual de daño que se va produciendo en las células externas del oído interno. Precisamente, Müller y su equipo (2010) consideran esperable que las exposiciones regulares a altos niveles sonoros de música por parte de los adolescentes causen daño irreversible en la audición a lo largo de los años. Sin embargo, debe destacarse que algunas personas presentan una vulnerabilidad especial frente a la exposición excesiva de ruido, mientras que otras se caracterizan por tolerar en mayor medida el impacto acústico excesivo sin daño auditivo evidente. El mecanismo subyacente de este fenómeno no se conoce en su totalidad, es por ello que investigaciones recientes se focalizan en el estudio de la función protectora coclear regulada por el Sistema Eferente Medial (SEM) en situaciones de riesgo para la salud auditiva (Maison \& Liberman, 2000; Hinalaf et al., 2011a; Hinalaf et al., 2011b).

En 1946, Rassmussen identificó un conjunto de fibras, llamado haz olivo-coclear cruzado, que se origina en la zona medial del complejo olivar superior y que cruza hacia la cóclea contralateral, a nivel del órgano de Corti del oído interno. Estas fibras son muy importantes debido a que por su intermedio, los centros regularizan la función coclear (Curet, 1988). Más tarde, Warr y Guinan (1979) describieron la existencia de dos subpoblaciones neuronales, una de ellas conformada por el SEM cuyas fibras, en su mayoría, se proyectan hacia las células ciliadas externas (CCE) de la cóclea contralateral.

En la actualidad, muchas de las posibles funciones del SEM son aún desconocidas. Sin embargo, la aparición de las otoemisiones acústicas (OAEs - Kemp, 1978), permitió realizar estudios específicos del oído interno, a través del registro de la actividad de las CCE. En 1990, las investigaciones iniciales de Collet y colaboradores incorporaron la supresión contralateral (SC) de OAEs para el estudio del efecto de la estimulación del SEM en las $\mathrm{CCE}$, principalmente en humanos. Si bien este estudio fue experimentado en los diversos tipos de OAEs, una de las más estudiadas ha sido la SC de las otoemisiones acústicas transitorias (TEOAEs).

La SC de las OAEs es un estudio relativamente nuevo, no existe a nivel internacional un acuerdo con respecto a la metodología de trabajo y a los criterios de aplicación para la técnica y análisis, ya que varían de una investigación a otra. Sin embargo, y a pesar de que aún no se conocen todas las posibles implicancias y aplicaciones clínicas de este estudio, el interés sobre el mismo y su valor diagnóstico se halla actualmente en pleno auge.

La importancia de nuevas investigaciones orientadas a profundizar el conocimiento sobre las funciones del SEM y la SC de las OAEs se basa en la hipótesis de que el SEM cumple una función moderadora de la contracción de las CCE del oído interno, prote- 
giendo al oído ante ruidos excesivos al reducir la capacidad del amplificador y mejorar la sensibilidad de la audición cuando el ambiente es ruidoso (Werner, 2001a). La función del SEM es inhibir la actividad de las CCE (De Ceulaer et al., 2001; Funchs, 2002; Ludwig et al., 2001; Oliveira, Massola de Fernandes \& Costa Filho, 2009; Valeiras, Dios, Porto \& Labella, 2005; Werner, 2006).

En Argentina no existen antecedentes de estudios sobre esta temática, a excepción del trabajo realizado por el equipo de Elgoyhen, en el que participan colaboradores de Estados Unidos (Taranda et al., 2009), que investigan el mecanismo protector del SEM a través de mutaciones genéticas en modelos animales, con resultados muy alentadores sobre la resistencia del oído ante ruidos intensos. De allí la importancia de profundizar sobre el funcionamiento de este sistema protector del oído en humanos y la posibilidad de relacionarlo con el deterioro prematuro de la audición que se observa en la actualidad, cada vez con mayor frecuencia, constituyendo un motivo de preocupación a nivel mundial.

Precisamente, esta problemática con respecto a la exposición a altos niveles sonoros de los adolescentes y el deterioro auditivo temprano condujo a plantear la investigación que se informa a fin de estudiar la dimensión psicosocial, para conocer los niveles de participación en actividades recreativas asociadas a altos niveles sonoros de música, y la dimensión auditiva, para conocer el funcionamiento del SEM, a través del estudio de la SC de las TEOAEs y los perfiles audiométricos, mediante la aplicación de la audiometría en los rangos convencional y extendido de alta frecuencia ${ }^{1}$.

1 La investigación se desarrolla en el marco de un Programa de Conservación y Promoción de la Audición en adolescentes implementado en el Centro de Investigación y Transferencia en Acústica (CINTRA).
Se planteó como objetivo analizar la posible relación entre la exposición general a música de los adolescentes durante su participación en actividades recreativas y el estado de la función auditiva conjuntamente con el estado del mecanismo de protección coclear.

\section{MetodologíA}

\section{PARTICIPANTES}

La muestra estuvo conformada por 91 alumnos (182 oídos) con edades entre 14 y 15 años y con predominio del sexo masculino, pertenecientes a dos escuelas técnicas (IPE Ms) de la ciudad de Córdoba. Se contó con el aval del Ministerio de Educación de la Provincia de Córdoba y la aprobación de las autoridades de ambos institutos educacionales. La participación de los adolescentes en el estudio fue voluntaria, debiendo contar con el consentimiento informado firmado por sus padres o tutores.

En la muestra se incluyeron adolescentes que tuvieran: TEOAEs presentes en ambos oídos, es decir, dentro de los parámetros considerados normales, Reproductibilidad $\geq 70 \%$ y una Relación Señal Ruido (SNR) $\geq 6 \mathrm{~dB}$ SPL en al menos tres de las frecuencias estudiadas $(1000,1500,2000,3000,4000) \mathrm{Hz}$ y oído medio sin alteraciones. Para descartar cualquier problema conductivo, se consideró la Timpanometría con Timpanograma tipo A, de acuerdo a la clasificación de Jerger (Medrano, C.R. \& Medrano, R.R. (2003).

\section{INSTRUMENTOS Y EQUIPOS DE MEDICIÓN}

1.- Dimensión psicosocial: Se administró el Cuestionario de Actividades Extraescolares (CAE) de Schuschke, Rudloff, Penk y su grupo de trabajo (1994) pertenecientes al Instituto de Medicina e Higiene de la Facultad de Medicina de la Universidad de Otto von Guericke de Magdeburgo (Alemania). El cuestionario fue adaptado a la población argentina por Serra y colaboradores (Serra et al., 2005), siguiendo la metodología de traducción di- 
recta e inversa recomendada en la literatura, consistente en la traducción al español, revisión sistemática del contenido del instrumento y discusión por el equipo de investigación, una traducción inversa a la lengua original alemana y un nuevo análisis de contenido del mismo.

El CAE está compuesto por 62 preguntas que permiten conocer en detalle la participación de los encuestados en cinco actividades recreativas: Escuchar música en el hogar, Interpretar instrumentos musicales y / o participar en grupos musicales, Asistir a conciertos en vivo, Usar reproductores personales de música y Asistir a lugares bailables. Para cada dimensión, el cuestionario permite evaluar la frecuencia de participación en cada actividad, la duración, antigüedad y nivel de exposición percibido. A partir del puntaje obtenido en cada actividad se obtuvo el Nivel de participación en las mismas, clasificándolo de acuerdo a una escala ordinal (No participa, Nivel bajo, Nivel medio y Nivel alto). Finalmente, la Exposición General a Música (EGM) se calculó a partir del puntaje obtenido para cada actividad y fue analizado en función de las siguientes categorías: Exposición baja, Exposición media, Exposición alta y Exposición muy alta.

2.- Dimensión auditiva: Se administró el Cuestionario de Estado Auditivo, construido ad hoc siguiendo los lineamientos aportados por la Norma ISO TC 43: CD 389-5, para conocer los antecedentes que pueden afectar la función auditiva y se realizaron los siguientes estudios:

- Otoscopía, para conocer el estado del canal auditivo, mediante el uso de un otoscopio clínico Heine, modelo Beta.

- Timpanometría, para determinar el estado del oído medio y su relación con los posibles problemas conductivos, usando un impedanciómetro automático Minitymp Kamplex MT 10 Interacoustics.

- Audiometría, en los rangos convencionales (250-8000) Hz y extendido de alta frecuencia (8000-16000) Hz, como método subjetivo, para determinar el umbral auditivo en toda la gama audible del individuo. Su aplicación se cumplimentó con las Normas
IRAM 4028-1 (1992), ISO 8253-1 (2010). Los saltos del estímulo auditivo de la audiometría fueron fijados en $3 \mathrm{~dB}$ HL para una mayor discriminación. De acuerdo a los resultados de la audiometría se dividió a los oídos estudiados en dos grupos: Normal: con umbrales auditivos menores o iguales a $18 \mathrm{~dB} \mathrm{HL}$ en cada una de las frecuencias analizadas en ambos rangos de frecuencias; Con descenso: con umbrales auditivos mayores de $18 \mathrm{~dB}$ HL en una o más frecuencias evaluadas. Para la realización de las audiometrías en ambos rangos de frecuencia se utilizaron un audiómetro Madsen Orbiter 922 y auriculares normalizados Senheiser HDA 200.

- Otoemisiones acústicas transitorias (TEOAEs), como método objetivo, para conocer el comportamiento mecánico de la cóclea y la $\mathrm{SC}$ de las TEOAEs, para conocer el funcionamiento del SEM y determinar presencia y ausencia de efecto supresor. El estudio consistió en:

Paso 1: aplicación de las TEOAEs y evaluación de un oído en forma homolateral ( $\sin$ estimulación acústica en el oído contralateral). Se utilizó un estímulo click con 260 presentaciones de estímulos, con una intensidad aproximada de $80 \mathrm{~dB}$ SPL, en modo no lineal, con nivel de rechazo de 47,3 dB SPL y con una estabilidad de estímulo mayor o igual a $85 \%$. Las frecuencias estudiadas fueron $(1000,1500,2000,3000,4000) \mathrm{Hz}$.

Paso 2: aplicación de las TEOAEs en el oído homolateral y al mismo tiempo aplicación de estimulación acústica en el oído contralateral (EAC), lo que permite conocer el funcionamiento del SEM. En las TEOAEs se utilizaron los mismos parámetros de medición utilizados en el Paso 1. Para la EAC se utilizó ruido blanco o banda ancha a una intensidad de $50 \mathrm{~dB}$ generado por el audiómetro.

De acuerdo a los resultados de la SC de las TEOAEs se dividió a los oídos estudiados en dos grupos: oídos con presencia de efecto supresor y con ausencia de efecto supresor.

Se consideró la presencia de efecto supresor cuando se producía una reducción de la respuesta luego de la EAC, es decir cuando la diferencia de los valores obtenidos en la respuesta total sin EAC y con EAC era positiva. 
A su vez, se consideró ausencia de efecto supresor en los casos de mantenerse o aumentarse la respuesta luego de la EAC, obteniendo un valor de cero $(0)$ o negativo. La ausencia de efecto supresor se considera una posible alteración del SEM y en la función que ejerce sobre la regulación de las células ciliadas externas.

Para la realización de las TEOAEs y de la SC de las TEOAEs, se utilizó un equipo para OAEs Otodynamics Ltd DP ECHOPORT ILO 292 USB II provisto con una sonda UGD TE+DPOAE Probe. Además se usó una sonda EAR TONE A3 para la EAC en el oído contralateral para evaluar la SC, provista por el audiómetro Madsen Orbiter 922, ya mencionado. A este conjunto se agregó una laptop con software de análisis clínico y gestión de datos llamado ILOV6, para el registro y realización de los estudios de las OAEs y dentro de ellas las TEOAEs.

\section{Procedimiento}

Para el estudio psicosocial se aplicó el CAE, de manera autoadministrada en las aulas y en un tiempo aproximado de 20 minutos, a grupos de 10 a 15 adolescentes por vez. Las pruebas para el estudio audiológico se llevaron a cabo en una cabina audiométrica móvil y acústicamente acondicionada, que cumplimenta con el ambiente exigido por la Normativa vigente -IRAM 4028-1 (1992) e ISO 8253-1 (2010)- en relación a su aislamiento sonoro del ruido exterior y a la absorción sonora interior.

La aplicación de las pruebas auditivas fue individual con una duración aproximada de 30-40 minutos por participante.

\section{ANÁLISIS ESTADÍSTICO}

Para el análisis estadístico se consideró como unidad de estudio al oído. Se realizaron los siguientes procedimientos:

1.- Confección de tablas de frecuencia y diagrama de barras con sus categorías para el análisis de la EGM.
2.- Análisis descriptivo para los datos de los umbrales auditivos y la respuesta total en las condiciones sin y con EAC.

3.- Aplicación del test $t$ para el estudio del nivel de significación del efecto supresor en relación a ambos oídos y de los umbrales auditivos en relación a los oídos.

4.- Aplicación del test $t$ apareado para la comparación de las diferencias de la respuesta total sin y con EAC.

5.- Aplicación del test t de Student para la comparación de las diferencias de los umbrales auditivos de cada frecuencia en los grupos normal y con descenso.

6.- Realización de tablas de contingencia para el análisis de asociación entre los niveles de EGM y los grupos con presencia y ausencia de efecto supresor.

En todos los casos se consideró un nivel de significación de $p<.05$.

Se utilizó el software estadístico InfoStat Versión 2015, desarrollado por un equipo de docentes-investigadores de la Facultad de Ciencias Agropecuarias de la Universidad Nacional de Córdoba (Di Rienzo et al., 2015).

\section{Resultados}

Dado que el análisis inferencial no mostró diferencias estadísticamente significativas entre oído izquierdo-derecho en el efecto supresor $(p=.45)$ y umbrales auditivos en las frecuencias evaluadas ( $p>.05)$, en los análisis posteriores no se realizó diferenciación entre ambos.

\section{SUPRESIÓN CONTRALATERAL DE LAS TEOAES Y EGM}

En la Tabla 1 se muestra la distribución en frecuencia y porcentaje de los oídos con presencia y ausencia de efecto supresor según las categorías de Exposición General a Música (EGM).

Sobre el total de oídos evaluados, se observa que la mayor concentración en las categorías alta y media de exposición (31 y $30 \%$, respectivamente) se produce en oídos 
con presencia de efecto supresor, mientras que en el grupo con ausencia de efecto supresor, la distribución fue similar en las categorías media, baja y alta. El análisis estadístico de la asociación entre presencia y ausencia de efecto supresor en relación a EGM no mostró resultados significativos $\left(\chi^{2}=2.06 ; p>.56\right)$. Posteriormente, se aplicó el test $t$ apareado para muestras relacionadas con el fin de analizar la existencia de diferencias de la respuesta total sin y con estimulación acústica contralateral (EAC) según las categorías de EGM (ver Tabla 2).

En todos los casos la diferencia de la respuesta total en las condiciones sin y con EAC fue estadísticamente significativa para cada una de las categorías de EGM. En los oídos con presencia de efecto supresor se observó una reducción de la respuesta total luego de la EAC, mientras en los oídos con ausencia de efecto supresor se manifestó aumento de respuesta total luego de la estimulación acústica. La categoría muy alta de EGM, en el grupo de ausencia de efecto supresor, no se visualiza por contar con dos casos para el análisis.

En la Figura 1 se muestra el efecto supresor con sus puntos promedios según las categorías de EGM.

La mayor concentración de oídos con presencia de efecto supresor se encuentra en las categorías media y alta; al comparar ambos grupos se observó menor magnitud del efecto supresor en la categoría alta. Sin embargo, la categoría baja es la que presenta menor magnitud en comparación al resto. En la Figura 1 no se muestran los puntos promedios referidos a la categoría muy alta debido a los pocos casos de ausencia de efecto supresor.

Considerando el mayor porcentaje de oídos en las categorías media y alta, se aplicó el test $t$ Student (ver Tabla 3) en los oídos con presencia de efecto supresor para estudiar las diferencias de la respuesta total en las condiciones sin y con EAC entre ambas categorías.

En general, los resultados mostraron que las categorías de EGM media y alta no influyeron en la respuesta total en ambas condiciones ( $\sin$ y con EAC: $p=.45$ y $p=.47$, respectivamente). Sin embargo, los oídos que pertenecen a la categoría alta de EGM y que tuvieron presencia de efecto supresor mostraron menor magnitud promedio de efecto supresor y disminución de la amplitud de la respuesta total en comparación con los oídos ubicados en la categoría media de EGM.

\section{SUPRESIÓN CONTRALATERAL DE LAS TEOAES Y AUDIOMETRÍA}

Los resultados de la muestra total mostraron que el mayor porcentaje de adolescentes, conformado por el 76\% (139 oídos), se concentró en el grupo normal caracterizado por presentar umbrales auditivos menores o iguales a $18 \mathrm{~dB} \mathrm{HL}$ en todas las frecuencias analizadas; mientras que el 24\% (43 oídos) se ubicó en el grupo con descenso, es decir, con umbrales auditivos mayores a $18 \mathrm{~dB}$ HL en una o más frecuencias evaluadas.

La Figura 2 muestra los umbrales auditivos promedio correspondientes a las frecuencias convencionales $(250$ a 8000$) \mathrm{Hz}$ y extendida de alta frecuencia (8000 a 16000) Hz pertenecientes a los grupos normal y con descenso.

La comparación de los perfiles audiométricos de ambos grupos muestra un desplazamiento de los umbrales auditivos en el grupo con descenso en las frecuencias superiores a $2000 \mathrm{~Hz}$.

Se aplicó el test $t$ Student para analizar las diferencias entre los umbrales auditivos de los grupos normal y con descenso.

Como puede observarse en la Tabla 4, los resultados mostraron diferencias estadísticamente significativas entre los grupos normal $y$ con descenso en todas las frecuencias a partir de $3000 \mathrm{~Hz}$. En $250 \mathrm{~Hz}, 500 \mathrm{~Hz}, 1000 \mathrm{~Hz}$ y $2000 \mathrm{~Hz}$ los umbrales de ambos grupos fueron similares $(p=.78 ; p=.08 ; p=.16 ; p=.96$, respectivamente).

La Figura 3 muestra que en los oídos con presencia de efecto supresor, este efecto fue mayor en el grupo normal, manifestando reducción de la respuesta total luego de la aplicación de la estimulación acústica en el oído contralateral, mientras que en el grupo con descenso se encontró que el efecto supresor era menor. En los oídos con ausencia de efecto supresor se observó un ligero aumento en 
el efecto supresor (6 oídos) en el grupo con descenso auditivo en comparación con el grupo normal (27 oídos).

Posteriormente se aplicó el test $t$ apareado para analizar las diferencias entre la respuesta total en las situaciones sin y con EAC de acuerdo a los grupos normal y con descenso. Como puede observarse en la Tabla 5, la diferencia entre los grupos normal y con descenso según presencia y ausencia de efecto supresor fue estadísticamente significativa en las condiciones sin y con EAC.

\section{Discusión}

El trabajo realizado aporta evidencia sobre la menor magnitud del efecto supresor y la disminución en la respuesta total de la SC de las TEOAEs en aquellos adolescentes que manifestaron descenso en los umbrales auditivos. A su vez, los adolescentes que se clasificaron en las categorías alta y baja de EGM manifestaron similares características en la $\mathrm{SC}$, es decir, que el efecto supresor y la respuesta total se encontraron disminuidos. Si bien estos hallazgos no presentaron diferencias estadísticas significativas, los resultados podrían estar indicando alteraciones en el funcionamiento del SEM en adolescentes con exposición alta a ruido no ocupacional y umbrales auditivos descendidos.

En la actualidad, la música constituye la principal fuente sonora a la que se exponen los adolescentes, ya sea escuchada en su propio hogar, en discotecas, en conciertos en vivo, en reuniones o a través de equipos personales reproductores de música (Serra et al., 1998). Cuando esta exposición se realiza a altos niveles sonoros y por tiempos prolongados, constituye un verdadero riesgo para la salud auditiva dado que puede ser causa de deterioro auditivo prematuro. Algunos autores han introducido una denominación específica para esta situación: pérdida auditiva inducida por música (Morata, 2007; Portnuff, Fligor \& Arehart; 2011). Desde el punto de vista psicosocial, los especialistas califican como comportamiento de riesgo a esa tendencia cada vez más acentuada de adoles- centes y jóvenes a exponerse a niveles sonoros que resultan peligrosos para la audición (Biassoni et al., 2011; Bohlin \& Erlandsson, 2007; Vogel, Brug, Hosli, van der Ploeg \& Raat, 2008, 2011; Vogel, Verschuure, van der Ploeg, Brug \& Raat, 2009; Widen, 2006; Widen \& Erlandsson, 2007).

Se estima que en dos décadas el número de jóvenes con exposición a ruido social se triplicará en comparación con la década de 1980, mientras que el ruido ocupacional habrá disminuido (Scientific Committee on Emerging and Newly Identified Health Risks [SCENIHR], 2008). Por su parte, la OMS en un comunicado emitido en el año 2015 informa que más de 1.100 millones de jóvenes en el mundo están en riesgo de sufrir pérdidas de audición a causa de usar en forma reiterada dispositivos electrónicos y auriculares a niveles sonoros más altos que los recomendados, mientras que ya hay más de 43 millones de jóvenes entre 12 y 35 años con discapacidades auditivas. En los países de ingresos medios y altos, casi el 50\% de los jóvenes de esa edad escuchan sus dispositivos electrónicos (MP3, teléfonos móviles y otros) a niveles no adecuados para la salud auditiva. Además, cerca del 40\% de esos mismos jóvenes están también expuestos a niveles excesivos de ruido en discotecas, bares y eventos deportivos. Estas declaraciones, junto con los resultados de numerosos estudios llevados a cabo a nivel mundial, muestran la gravedad de la problemática planteada la que a su vez nos concierne a todos. Este panorama pone de manifiesto la necesidad de contar con pruebas específicas que permitan conocer en forma temprana el estado de los sistemas que hacen a la función auditiva a fin de poder predecir la mayor o menor vulnerabilidad del oído ante exposiciones a altos niveles sonoros y de este modo actuar preventivamente.

Los resultados de la investigación realizada están mostrando que desde temprana edad, 14 y 15 años, un alto porcentaje de los adolescentes evaluados se ubica en las categorías media y alta de participación en actividades recreativas caracterizadas por música. Diferentes estudios muestran que la participación en ese tipo de actividades, y 
por ende la exposición a música, va aumentando con el paso del tiempo, con el riesgo de un daño acumulativo de la audición (Biassoni et al., 2014; Jofre et al., 2009; Müller et al., 2010; Serra et al., 2014; Zenker et al., 2001).

Al relacionar las categorías de EGM, en las que se ubican los participantes con los resultados de la SC de las TEOAEs se observó que los oídos con presencia de efecto supresor mostraron una reducción estadísticamente significativa de la respuesta total luego de la EAC, ubicándose la mayoría de estos oídos dentro de las categorías alta y media de EGM. En cambio, los oídos con ausencia de efecto supresor evidenciaron un incremento estadísticamente significativo, en la respuesta total luego de la aplicación acústica. A su vez, los oídos con ausencia de efecto supresor se ubicaron, en porcentajes similares, dentro de las categorías media, baja y alta de EGM; además presentaron una distribución menor que los oídos con presencia de efecto supresor y sus porcentajes fueron semejantes en las tres categorías. Valeiras y colaboradores (2005) señalan que la ausencia de efecto supresor se puede considerar como un hallazgo patológico, indicativo de alteración en el SEM. Otro aporte realizado por Muñiz (2005) se refiere a que la ausencia de efecto supresor contralateral se relacionaría con la existencia de fatiga auditiva por lo cual el oído podría ser susceptible de ser lesionado por exposición a ruido.

Como se ha mencionado, algunos investigadores (Rajan, 2000; Valeiras et al., 2005; Werner, 2001) sugieren que el SEM tendría un rol importante en la protección ante la sobrestimulación acústica. Por lo tanto, se podría hipotetizar que un oído con presencia de efecto supresor tendería a estar más protegido ante la exposición a altos niveles sonoros, mientras que los oídos con ausencia de efecto supresor podrían ser más vulnerables ante la exposición a ruido.

Si bien los oídos con presencia de efecto supresor estarían protegidos por la regulación del SEM en situaciones de ruido, al comparar las categorías de EGM no se observaron diferencias estadísticamente significativas entre los grupos, pero sí una menor magnitud (en promedio) de efecto supresor en las cate- gorías alta y baja de EGM. Estos oídos podrían considerarse un grupo de riesgo auditivo si mantienen o aumentan su exposición al ruido en un futuro. A su vez, los resultados actuales que muestran disminución de la magnitud del efecto supresor, podrían estar indicando disminución o alteración en el funcionamiento del SEM. En ese caso, el SEM no lograría proteger adecuadamente a las CCE del oído interno durante la exposición a sonidos intensos, lo cual se haría cada vez más evidente si a futuro la exposición a ruido continúa y / o se acentúa y a su vez la magnitud del efecto supresor continúa decreciendo o se produce ausencia del mismo.

Cabe señalar que la categoría baja de EGM, según sus respuestas al Cuestionario de Actividades Extraescolares, es la que en promedio presenta menor magnitud de efecto supresor en comparación a las categorías media y alta. Aunque este grupo no se expone todavía a intensidades elevadas de música, surge la hipótesis de que quizás estos oídos podrían ser caracterizados al presente como oídos sensibles a la sobreestimulación, y si en el futuro ese nivel de exposición se incrementa, siendo insuficiente la protección auditiva, pueda ser causa de un deterioro auditivo prematuro.

Otras variables consideradas fueron los umbrales auditivos obtenidos de la audiometría convencional y extendida de alta frecuencia, a partir de los cuales se clasificaron los oídos en normales con descenso. Los resultados mostraron que el mayor porcentaje de oídos se concentró en el grupo normal (77\%), mientras que el $23 \%$ se ubicó en el grupo con descenso. Los umbrales auditivos en el grupo con descenso mostraron un desplazamiento, con diferencia estadísticamente significativa en relación con los umbrales auditivos del grupo normal, en la mayoría de las frecuencias, a excepción de las frecuencias 250, 500, 1000 y $2000 \mathrm{~Hz}$ pertenecientes al rango convencional.

Los resultados de los oídos con presencia de efecto supresor mostraron que éste fue menor en el grupo con descenso y también fue menor la respuesta total en las condiciones sin y con EAC en comparación con el grupo normal. Si bien las diferencias entre 
ambos grupos no fueron estadísticamente significativas, esta disminución puede llegar a ser una manifestación incipiente de un problema en el funcionamiento del SEM en el grupo con descenso en los umbrales auditivos. En un trabajo realizado por Carvallo (2002) se encontró una tendencia de menor reducción en la magnitud de supresión en las TEOAEs y en la respuesta total, cuando los umbrales en las altas frecuencias (9000 a $20000 \mathrm{~Hz}$ ) se encontraban desplazados. Sin embargo, no se obtuvieron diferencias estadísticamente significativas en la magnitud de supresión con el grupo sin alteración en las altas frecuencias. Por otro lado, Sliwinska-Kowalska y Kotylo (2002) observaron que la supresión de las OAEs resultó moderadamente disminuida y en otros casos casi nula en el grupo de sujetos expuestos a ruido en comparación a los no expuestos. Los autores sugieren que la supresión contralateral de las OAEs podría brindar indicios de detección temprana de daño auditivo después de la exposición a ruido.

Los grupos normal y con descenso en general no mostraron variaciones significativas con respecto al efecto supresor y a la respuesta total con la EAC en los oídos con ausencia de efecto supresor. Sin embargo, los resultados indican que el grupo con descenso (6 oídos) presentó un ligero aumento en la respuesta total sin y con EAC en comparación con el grupo normal (27 oídos). De acuerdo a los resultados mencionados se podría inferir que en los oídos con ausencia de efecto supresor se produjo una menor actividad en la regulación sobre las CCE con la aplicación de la EAC; por lo tanto, esto podría estar indicando que en estos oídos el mecanismo reflejo no tendría una acción protectora óptima ante la exposición a ruido. Es por ello que se plantea la cuestión de que si los oídos con ausencia de efecto supresor, pertenecientes al grupo normal al momento del estudio, en el futuro pueden presentar un desplazamiento temprano de sus umbrales auditivos, al suponer que su mecanismo de protección ante exposiciones a altos niveles sonoros puede encontrarse afectado a causa de alguna posible alteración del SEM. Cabe recordar que los resultados presentados corresponden a adolescentes de sólo 14 / 15 años de edad y que, según lo indican diferentes autores, la mayoría de los adolescentes acentúan con la edad su exposición al denominado ruido no ocupacional o ruido social, aumentando el riesgo de daño auditivo (Biassoni et al., 2008; Jofre et al., 2009; Zenker et al., 2001).

Los resultados obtenidos con adolescentes de 14 / 15 años, a partir de un diseño de tipo transversal, demuestran la importancia de realizar un seguimiento de esos adolescentes para conocer a través del tiempo el comportamiento de las variables consideradas en el trabajo realizado. Si bien en la literatura general la mayoría de los estudios son de tipo transversal, hay autores que se inclinan por estudios a largo plazo, como es el caso de Wagner y colaboradores (2005) quienes sugieren este tipo de estudios en futuras investigaciones a fin de poder evaluar la posible asociación entre la actividad del SEM y el desplazamiento permanente del umbral auditivo en los seres humanos.

A su vez, otros autores como Müller y su equipo (2010) señalan la necesidad de continuar investigando sobre las funciones del SEM, en particular sobre el papel que este sistema desempeña como predictor de la susceptibilidad individual al ruido en los casos de pérdida de audición permanente.

Profundizar sobre la exposición a ruido social en adolescentes y la especificidad de la prueba en relación con los cambios que se van produciendo en los umbrales auditivos permitirá aumentar la fiabilidad y validez del análisis de la SC de las TEOAEs como un medio para comprender la inhibición del SEM. De esta manera y en adición a futuros trabajos, se puede contribuir al diagnóstico para el conocimiento de su mecanismo de protección coclear y consecuente sensibilidad individual ante la exposición a ruido para actuar tempranamente en la prevención de las hipoacusias inducidas por ruido y favorecer a la salud auditiva. El avance en esta temática permitirá contribuir en futuras acciones tendientes a actuar preventivamente sobre la vulnerabilidad auditiva de los jóvenes de manera temprana, antes que la pérdida auditiva se encuentre en 
estadios irreversibles, y a su vez, advertir sobre los hábitos recreativos relacionados con la participación en actividades referidas a la escucha de música a altos niveles sonoros. En este sentido, la investigación realizada muestra la importancia de implementar un Screening Psico-Auditivo destinado a detectar los comportamientos que pueden afectar la audición y predecir de manera temprana la vulnerabilidad auditiva a través de la evaluación del estado del SEM, a fin de contribuir en la prevención y promoción de la salud auditiva.

\section{Conclusiones}

Se considera de importancia destacar que la supresión contralateral (SC) de las otoemisiones acústicas (OAEs) es un estudio relativamente nuevo y por esta razón tanto su metodología de trabajo como los criterios para la técnica de aplicación y análisis aún no presentan uniformidad en las investigaciones a nivel internacional. A ello se agrega la escasez de equipamiento clínico para estudiar el SEM con los distintos modos de estimulación requeridos, todo lo cual hace difícil, al momento, poder establecer generalidades para la aplicación de la técnica y como paso siguiente lograr la normatización de la prueba.

Los resultados obtenidos en el trabajo descripto permiten concluir que desde temprana edad la mayoría de los adolescentes participantes tienen una exposición a música que se ubica dentro de las categorías media y alta, según sus respuestas al Cuestionario de Actividades Extraescolares aplicado, exposición que puede llegar a incrementarse con el tiempo. Si bien esos niveles de exposición no influyeron estadísticamente sobre el efecto supresor y la respuesta total en las condiciones sin y con EAC, se observó que en los oídos con presencia de efecto supresor y ubicados en la categoría alta de EGM presentaron menor magnitud del efecto y a su vez, disminución de la amplitud de la respuesta total en las condiciones sin y con EAC. Aunque esta misma situación se observó en los oídos ubicados en la categoría baja de EGM. Teniendo en cuenta que la ausencia de efecto supresor se considera una posible alteración del SEM y en su mecanismo protector coclear ante el ruido, el resultado obtenido que muestra menor magnitud del efecto en ambas categorías (alta y baja) de EGM sugiere que esos oídos serían oídos vulnerables si la exposición a altos niveles sonoros se mantiene o se incrementa en el futuro, es decir oídos más propensos a sufrir daño auditivo.

El otro resultado a destacar es el descenso de los umbrales auditivos observado en un grupo de los adolescentes participantes. Si bien la SC de las TEOAEs no presentó diferencia estadísticamente significativa entre los grupos normal y con descenso de los umbrales auditivos, en este segundo grupo, con presencia de efecto supresor, se observó un menor efecto y menor reducción de la respuesta total al aplicar la EAC en relación al primer grupo, o sea al grupo normal.

De los resultados obtenidos cabe señalar la menor magnitud de efecto supresor y la disminución en la respuesta total en relación con el descenso de los umbrales auditivos encontrados en oídos que se ubican en las categorías alta y baja de EGM. Estos resultados confirman la necesidad de promover futuros trabajos para dar continuidad a las investigaciones sobre la SC de las TEOAEs, destacando que en Argentina no existen al momento antecedentes referidos a la temática. Sería conveniente dar continuidad al tema con estudios a largo plazo que permitan conocer el funcionamiento del SEM, como mecanismo protector de la audición, en relación con el comportamiento de riesgo auditivo durante el período de la adolescencia. Esto haría posible el aporte de información destinada a comprobar la hipótesis que postula que la ausencia de la SC contribuiría en la identificación temprana de la sensibilidad o vulnerabilidad auditiva en los adolescentes expuestos a dosis elevadas de ruido no ocupacional. De probarse esta hipótesis, la SC brindaría su utilidad diagnóstica con la posibilidad de ser incluida en Programas de Conservación y Promoción Auditiva en adolescentes. 


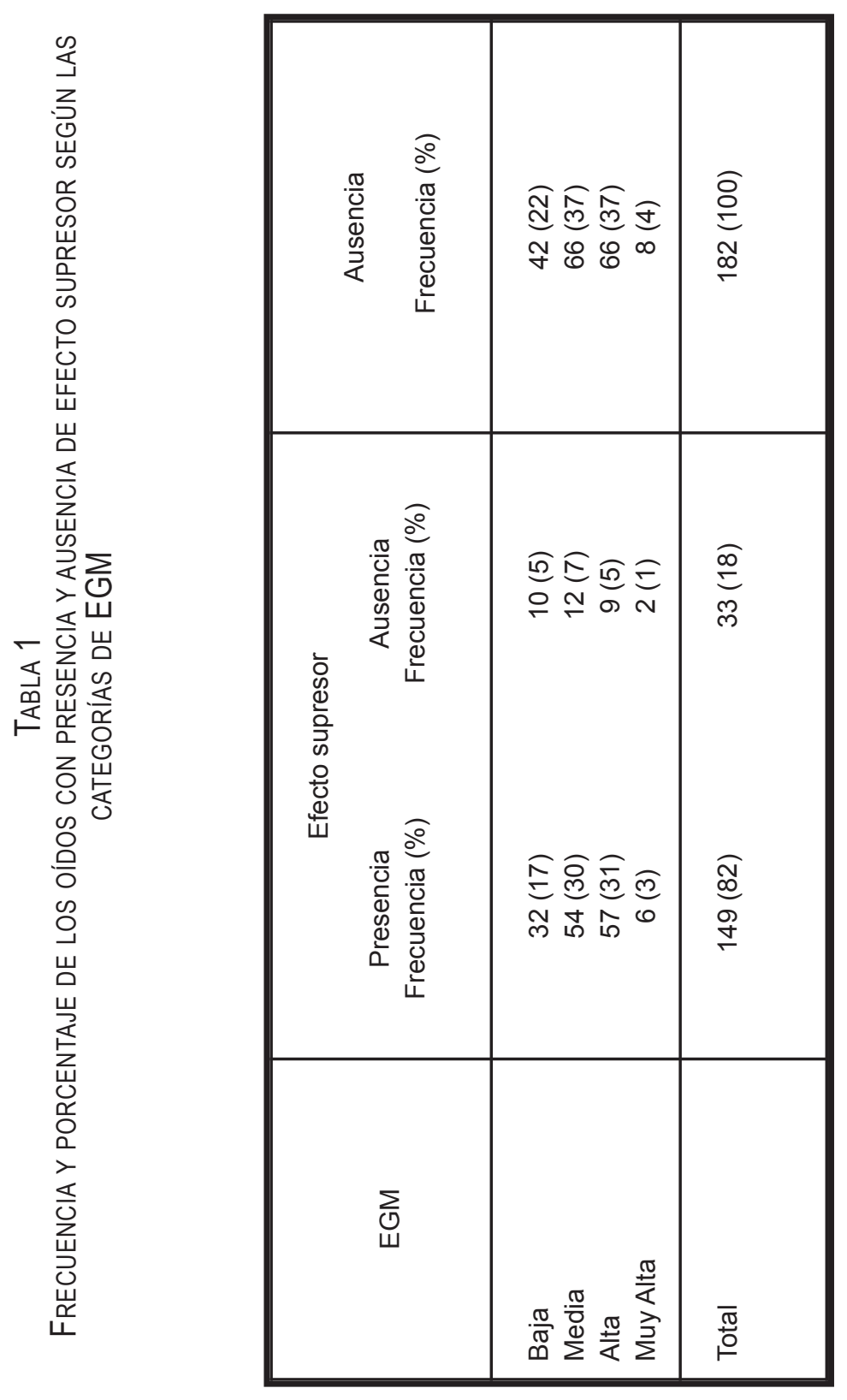


TABLA 2

DIFERENCIAS ENTRE LA RESPUESTA TOTAL "SIN" Y "CON" EAC CORRESPONDIENTE A LA PRESENCIA Y AUSENCIA DE EFECTO SUPRESOR SEGÚN LAS CATEGORÍAS DE EGM

\begin{tabular}{|c|c|c|c|c|c|c|}
\hline \multicolumn{7}{|c|}{ Presencia de Efecto Supresor } \\
\hline EGM & $n$ & $\begin{array}{c}M \\
\sin E A C\end{array}$ & $\begin{array}{c}M \\
\text { con EAC }\end{array}$ & Diferencia & $t$ & $\begin{array}{c}p \\
(p<.05)\end{array}$ \\
\hline $\begin{array}{l}\text { Baja } \\
\text { Media } \\
\text { Alta } \\
\text { Muy Alta }\end{array}$ & $\begin{array}{c}32 \\
54 \\
57 \\
6\end{array}$ & $\begin{array}{l}12.24 \\
13.74 \\
13.10 \\
13.52\end{array}$ & $\begin{array}{l}11.28 \\
12.63 \\
12.01 \\
12.73\end{array}$ & $\begin{array}{l}.96 \\
1.12 \\
1.09 \\
.78\end{array}$ & $\begin{array}{c}8.67 \\
13.13 \\
12.55 \\
3.94\end{array}$ & $\begin{aligned}<.0001 \\
<.0001 \\
<.0001 \\
.011\end{aligned}$ \\
\hline & \multicolumn{6}{|c|}{ Ausencia de Efecto Supresor } \\
\hline EGM & $n$ & $\begin{array}{c}M \\
\sin E A C\end{array}$ & $\begin{array}{c}M \\
\text { con EAC }\end{array}$ & Diferencia & $t$ & $(p<.05)$ \\
\hline Baja & 10 & 11.58 & 11.81 & -.23 & -4.12 & .0026 \\
\hline Media & 12 & 12.49 & 12.84 & -.35 & -5.87 & .0001 \\
\hline Alta & 9 & 13.53 & 13.91 & -.38 & -4.65 & .0016 \\
\hline
\end{tabular}

TABLA 3

DifERENCIAS dE LA RESPUESTA TOTAL ENTRE LAS CATEGORÍAs Alta y MEDIA EN AMBAS CONDICIONES DE LOS OÍDOS CON PRESENCIA DE EFECTO SUPRESOR

\begin{tabular}{|c|c|c|c|c|c|c|c|c|c|}
\hline Condición & Grupo & Grupo & $n$ & $n$ & $\begin{array}{c}\text { Respuesta } \\
\text { total } \\
M(1)\end{array}$ & $\begin{array}{c}\text { Respuesta } \\
\text { total } \\
M(2)\end{array}$ & Diferencia & $t$ & $\begin{array}{c}p \\
(2)\end{array}$ \\
\hline Sin EAC & Alta & Media & 57 & 54 & 13.1 & 13.74 & -.64 & -.75 & .4542 \\
Con EAC & Alta & Media & 57 & 54 & 12.01 & 12.63 & -.62 & -.72 & .4741 \\
\hline
\end{tabular}


TABLA 4

DIFERENCIAS ENTRE LOS UMBRALES AUDITIVOS DE LOS GRUPOS NORMAL Y CON DESCENSO SEGÚN FRECUENCIA

\begin{tabular}{|c|c|c|c|c|c|c|c|}
\hline $\begin{array}{c}\text { Frecuencia } \\
(\mathrm{Hz})\end{array}$ & $\begin{array}{c}\text { Con des- } \\
\text { censo } \\
(n)\end{array}$ & $\begin{array}{c}\text { Normal } \\
(n)\end{array}$ & $\begin{array}{c}M \text { Con } \\
\text { descenso } \\
(\mathrm{dB} \mathrm{HL})\end{array}$ & $\begin{array}{c}M \\
\text { Normal } \\
(\mathrm{dB} \mathrm{HL})\end{array}$ & $\begin{array}{c}\text { Diferencia } \\
(\mathrm{dB} \mathrm{HL})\end{array}$ & $t$ & $p<.05$ \\
\hline 250 & 43 & 139 & 5.23 & 5.03 & .20 & .271 & .7865 \\
500 & 43 & 139 & 5.86 & 4.27 & 1.59 & 1.796 & .0777 \\
1000 & 43 & 139 & 1.47 & .41 & 1.06 & 1.404 & .1622 \\
2000 & 43 & 139 & .35 & .30 & .05 & .050 & .9601 \\
3000 & 43 & 139 & 8.65 & 6.21 & 2.44 & 2.373 & .0209 \\
4000 & 43 & 139 & 10.33 & 6.35 & 3.98 & 2.691 & .0097 \\
6000 & 43 & 139 & 9.21 & 4.73 & 4.48 & 3.229 & .0021 \\
8000 & 43 & 139 & 3 & .52 & 2.48 & 2.323 & .0213 \\
9000 & 43 & 139 & 7.26 & 3.11 & 4.15 & 3.282 & .0018 \\
10000 & 43 & 139 & 7.26 & 2.81 & 4.45 & 3.190 & .0023 \\
11200 & 43 & 139 & 12.42 & 4.32 & 8.10 & 4.374 & .0001 \\
12500 & 43 & 139 & 13.4 & 2.20 & 11.19 & 5.408 & $<.0001$ \\
14000 & 43 & 139 & 20.65 & .56 & 20.09 & 8.243 & $<.0001$ \\
16000 & 43 & 139 & 24 & -1.12 & 25.12 & 10.358 & $<.0001$ \\
& & & & & & & \\
\hline
\end{tabular}

TABLA 5

DIFERENCIAS DE RESPUESTA TOTAL SIN Y CON EAC EN CADA GRUPO NORMAL Y CON DESCENSO SEGÚN PRESENCIA Y AUSENCIA DE EFECTO SUPRESOR

\begin{tabular}{|l|c|c|c|c|c|c|}
\hline \multicolumn{1}{|c|}{ Grupo } & $n$ & $\begin{array}{c}M \\
\sin \text { EAC }\end{array}$ & $\begin{array}{c}M \\
\text { con EAC }\end{array}$ & Diferencia & $t$ & $p<.05$ \\
\hline Normal (Presencia de efecto supresor) & 112 & 13.49 & 12.4 & 1.09 & 19.28 & $<.0001$ \\
$\begin{array}{l}\text { Con descenso (Presencia de efecto su- } \\
\text { presor) }\end{array}$ & 37 & 12.19 & 11.22 & .97 & 8.09 & $<.0001$ \\
Normal (Ausencia de efecto supresor) & 27 & 12.04 & 12.37 & -.33 & -7.29 & $<.0001$ \\
$\begin{array}{l}\text { Con descenso (Ausencia de efecto su- } \\
\text { presor) }\end{array}$ & 6 & 13.32 & 13.60 & -.28 & -4.03 & .01 \\
\hline
\end{tabular}


Efecto supresor según CATEgorías de Exposición General a Música (EGM)

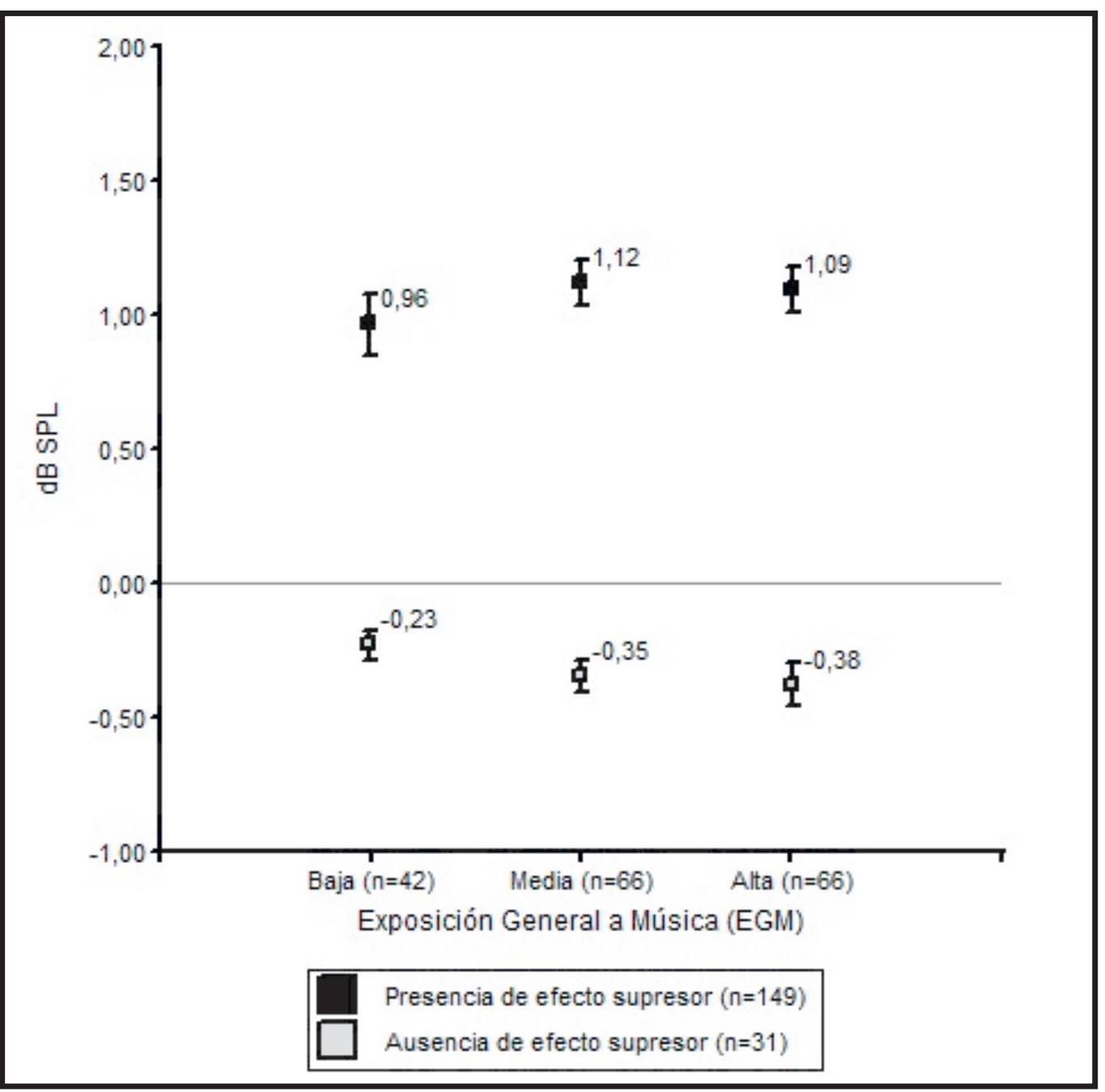




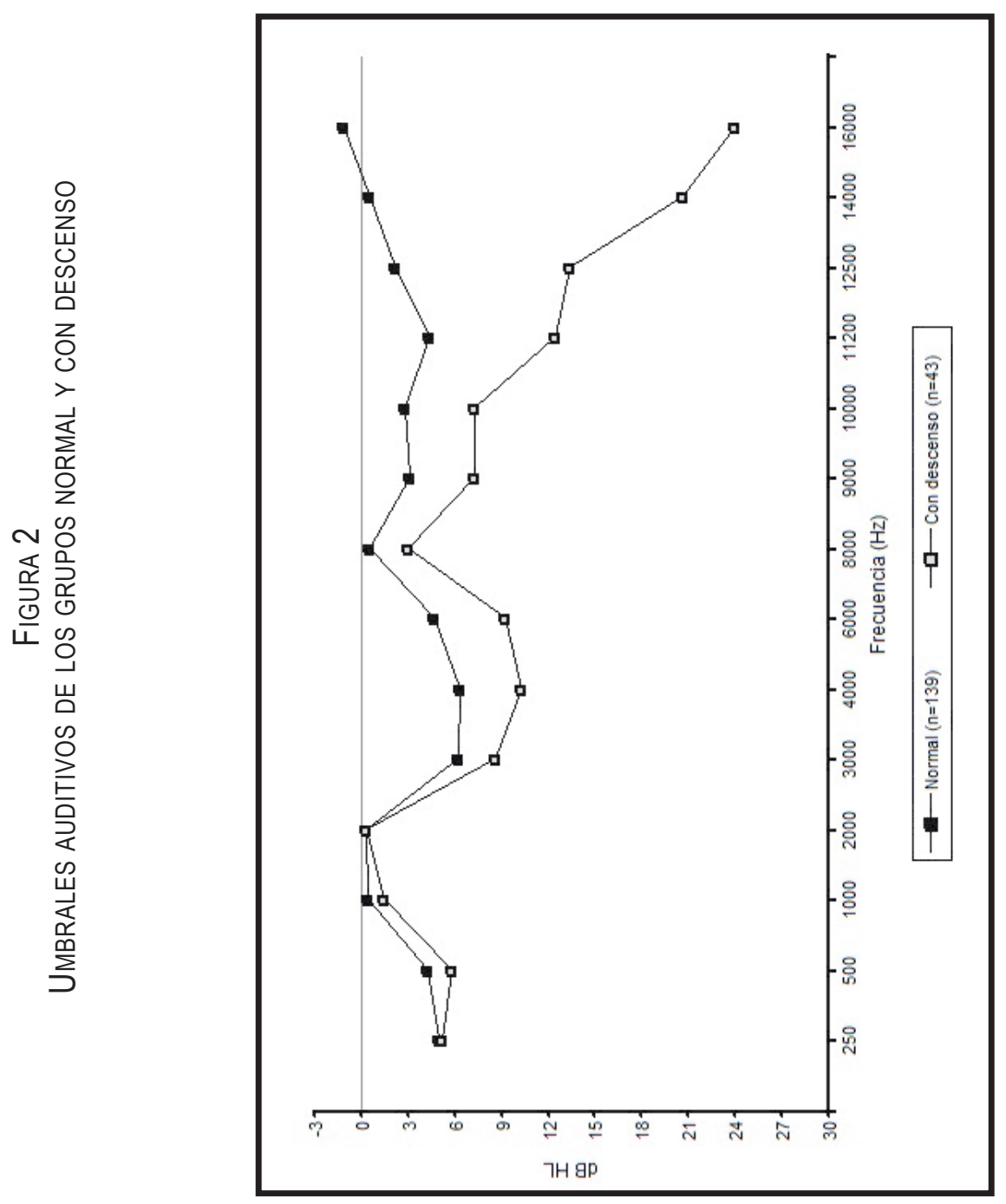


FIGURA 3

EFECTO SUPRESOR SEGÚN GRUPOS NORMAL Y CON DESCENSO

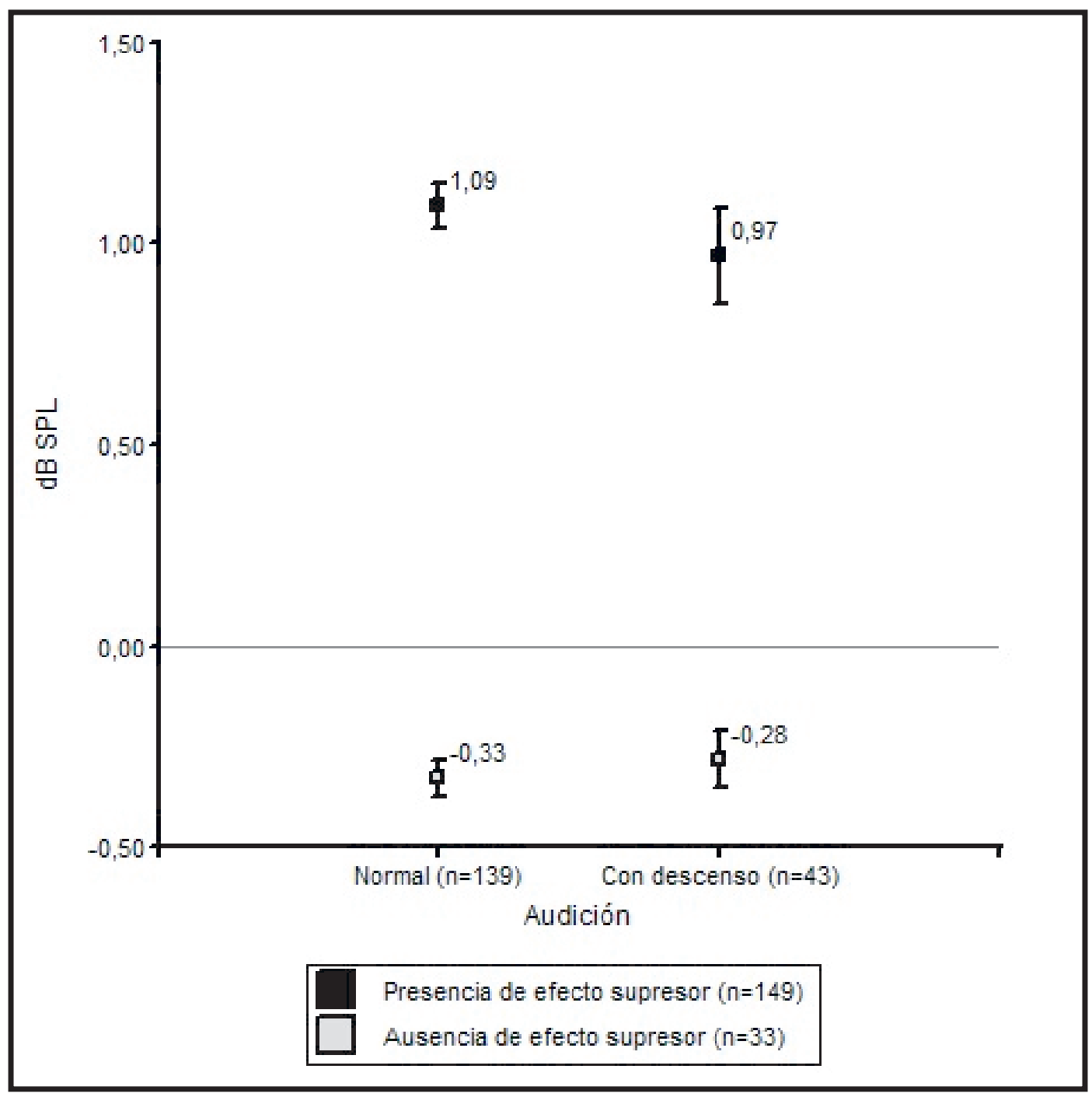




\section{REFERENCIAS BIBLIOGRÁFICAS}

Abraham, M., Hinalaf, M., Pavlik, M., Biassoni, E.C., Serra, M.R., Joekes, S., Righetti, A., Yacci, M.R. \& Pérez Villalobo, J. (2011). Deterioro auditivo por exposición a música en adolescentes argentinos [Hearing impairment for noise exposure in young argentinean]. Trabajo presentado en el $42^{\circ}$ Congreso Español de Acústica, Encuentro Ibérico de Acústica-Tecniacústica. Sociedad Acústica Española. Cáceres, España.

Biassoni, E.C., Serra, M.R., Hinalaf, M., Abraham, M., Pavlik, M., Pérez Villalobo, J., Curet, C., Joekes, S., Yacci, M.R. \& Righetti, A. (2014). Hearing and loud music exposure in a group of adolescents at the ages of 14-15 and 17-18. Noise \& Health, 16(72), 331-341. http://doi.or g/10.4103/1463-1741.140515

Biassoni, E.C., Serra, M.R., Pavlik, M., Hinalaf, M., Curet, C., Pérez Villalobo, J., Abraham, M., Gauchat, S., Joekes, S. \& Righetti, A. (2011). Programa de Conservación y Promoción de la Audición en la Etapa Adolescente: Primeros resultados [Conservation and Promotion Program of Hearing on Adolescent Stage: First results]. En M.C. Richaud \& V. Lemos (Eds), Compendio de investigaciones actuales en psicología y ciencias afines (pp. 57-503). Libertador San Martín: Universidad Adventista del Plata.

Biassoni, E.C., Serra M.R., Pérez Villalobo J., Joekes S. \& Yacci M.R. (2008). Hábitos recreativos en la adolescencia y salud auditiva [Recreational habits among adolescents and auditory health]. Revista Interamericana de Psicología, 42(2), 257-271.

Blair, J.C., Hardegree, D. \& Benson, P.V. (1996). Necessity and effectiveness of a hearing conservation program for elementary students. Journal of Educational Audiology, 4, 12-16.

Bohlin, M. \& Erlandsson, S. (2007). Risk behavior and noise exposure among adolescent. Noise \& Health, 9, 55-63.
Carvallo, R.M. (2002). Audição em alta freqüência: Repercussões no reconhecimento de fala no ruído e nas emissões otoacústicas [High frequency hearing: Reprercussions on speech in noise recognition and otoacoustic emission]. Tesis para obtener el título de Profesor Titular. Facultad de Medicina de la Universidad de San Pablo. Brasil.

Chung, J.H., Des Roches, C.M., Meunier, J. \& Eavey, R.D. (2005). Evaluation of noise-induced hearing loss in young people using a webbased survey technique. Pediatrics, 115(4), 861-867. http://doi.org/10.1542/peds.2004-01 73

Collet, L., Kemp, D.T., Veuillet, E., Duclaux, R., Moulin, A. \& Morgon, A. (1990). Effect of contralateral auditory stimuli on active cochlear micro-mechanical properties in human subjects. Hearing Research, 43, 251-61. http://doi.org/ 10. 016/0378-5955(90)90232-E

Curet, C. (1988). E.R.A. Audiometría por respuestas eléctricas. Potenciales precoces auditivos [Audiometry electrical responses. Early auditory potentials]. Buenos Aires, Argentina: CTM Servicios Bibliográficos S.A.

De Ceulaer, G., Yperman, M., Daemers, K., Van Driessche, K., Somers, T., Officierss, F.E. \& Govaerts, P.J. (2001). Contralateral suppression of transient evoked otoacoustic emission: Normative data for a clinical test set-up. Otology \& Neurotology, 22, 350-355. http://doi.org/10.109 7/00129492-200105000-00013

Di Rienzo, J.A., Casanoves, F., Balzarini, M.G., González, L., Tablada, M. \& Robledo, C.W. (2015). InfoStat versión 2015. Grupo InfoStat. FCA, Universidad Nacional de Córdoba, Argentina. Recuperado el 26 de mayo de 2015 de http://www.infostat.com.ar

Folmer, R.L., Griest, S.E. \& Martin, W.H. (2002). Hearing conservation education programs for children: A review. Journal of School Health, 72, 51-57. http://doi.org/10.1111/j.1746-1561. 2002.tb06514.x 
Funchs, P. (2002). The synaptic physiology of cochlear hair cells. Audiology and Neurotology, 7, 40-44. http://doi.org/10.1159/000046862

Hinalaf, M., Biassoni, E.C., Serra, M.R., Pavlik, M.L., Curet, C., Abraham, M., Pérez Villalobo, J., Joekes, S., Righetti, A. \& Yacci, M.R. (2011c). La vía eferente medial como mecanismo protector de la audición [The medial efferent system as a protective mecanism of hearing]. Revista Mecánica Computacional, 30, 3157-3166.

Hinalaf, M., Pavlik, M.L., Biassoni, E.C., Serra, M.R., Curet, C.A., Abraham, M., Joekes, S., Yacci, M.R. \& Righetti, A. (2011a). Estudio sobre la supresión contralateral de las otoemisiones acústicas transitorias, umbrales auditivos y hábitos recreativos en adolescentes [Study about suppression of transient evoked otoacoustic emissions, hearing thresholds and recreational habits in adolescents]. Revista Areté Fonoaudiología, 11, 55-69.

Hinalaf, M., Pavlik, M. L., Serra, M.R., Curet, C., Joekes, S. \& Yacci, M.R. (2011b). Hábitos recreativos y sensibilidad auditiva en adolescentes [Recreational habits and hearing sensitivity in adolescents]. En M.C. Richaud \& V. Lemos (Eds.), Psicología y otras ciencias del comportamiento (pp. 505-523). Entre Ríos, Argentina: Universidad Adventista del Plata.

Jofre, P.D., De la Paz, F.P., Platzer, L.M., Anabalón, J.L., Grasset, E.E. \& Barnafi, R.N. (2009). Evaluación de la exposición a ruido social en jóvenes chilenos [Assessment of social noise exposure in Chilean youth]. Revista de Otorrinolaringología y Cirugía de Cabeza y Cuello, 69(1), 23-28. http://doi.org/10.4067/S0718-48 162009000100005

IRAM 4028- (1992). Audiometría tonal. Métodos básicos de prueba [Audiometry. Basic testing methods]. Instituto Argentino de Normalización y Certificación.
ISO 8253-1 (2010). Acoustics - Audiometric test methods - Part 1: Pure-tone air and bone conduction audiometry. International Organization for Standardization.

Jofre, P.D., De la Paz, F.P., Platzer, L.M., Anabalón, J.L., Grasset, E.E. \& Barnafi, R.N. (2009). Evaluación de la exposición a ruido social en jóvenes chilenos [Assessment of social noise exposure in Chilean youth]. Revista de Otorrinolaringología y Cirugía de Cabeza y Cuello, 69(1), 23-28. http://doi.org/10.4067/S0718-48 162009000100005

Kemp, D.T. (1978). Stimulated acoustic emissions from within the human auditory system. Journal of the Acoustical Society of America, 64, 1386-1391. http://doi.org/10.1121/1.382104

Ley Nacional 79587/72 - Higiene y seguridad en el trabajo [Health and security at work] - Decreto Reglamentario 351/79 y Resolución modificatoria 295/03. Buenos Aires, Argentina.

Ludwig, J., Oliver, D., Frank, G., Klöcker, N., Gummer, A.W. \& Fakler, B. (2001). Reciprocal electromechanical properties of rat prestin: The motor molecule from rat outer hair cells. Proceedings of the National Academy of Sciences, 98, 4178-4183. http://doi.org/10.1073/ pnas. 071613498

Maison, S.F. \& Liberman, M.C. (2000). Predicting vulnerability to acoustic injury with a noninvasive assay of olivocochlear reflex strength. The Journal of Neuroscience, 20, 4701-4707.

Medrano, C.R. \& Medrano, R.R. (2003). Neurootofisiología y audiología clínica [Otophysiology and clinical audiology]. México: McGraw-Hill.

Meyer-Bisch, C. (1996). Epidemiological evaluation of hearing damage related to strongly amplified music (personal cassette players, discotheques, rock concerts)-high definition audiometric survey on 1364 subjects. Audiology, 35, 121-142. http://doi.org/10.3109/0020609 9609071936 
Morata, T.C. (2007). Young people: Their noise and music exposures and the risk of hearing loss. International Journal of Audiology, 46(3), 111-112. http://doi.org/10.1080/149920206011 03079

Morioka, I., Luo, W.Z., Miyashita, K., Takeda, S., Wang Y.X. \& Li S.C. (1996). Hearing impairment among young Chinese in a rural area. Public Health, 110, 293-297. http://doi.org/10. 1016/S0033-3506(96)80092-8

Müller, J., Dietrich, S. \& Janssen, T. (2010). Impact of three hours of discotheque music on pure-tone thresholds and distortion product otoacoustic emissions. The Journal of the Acoustical Society of America, 128, 1853-1869. http: //doi.org/10.1121/1.3479535

Muñiz, J.F. (2005). Estudio de la correlación existente entre el efecto supresor contralateral y la fatiga auditiva mediante otoemisiones acústicas transitorias [Study about the relations between contralateral supresor effect and auditory fatigue with transitory acoustic emissions]. Tesis Doctoral no publicada. Facultad de Medicina, Universidad de Valencia.

National Institutes of Health (2000). Healthy people 2010: focus area 28. Vision and hearing. Office of Disease Prevention and Health Promotion. USA: Department of Health and Human Services.

Niskar, A.S., Kieszak, S.M., Holmes, A.E., Esteban, E., Rubin, C. \& Brody D.J. (2001). Estimated prevalence of noise-induced hearing threshold shifts among children 6 to 19 years of age: The Third National Health and Nutrition Examination Survey, 1988-1994, United States. Pediatrics, 108, 40-43.

Oliveira, J.R., Massola de Fernandes, J.C. \& Costa Filho, O.A. (2009). Influência da idade na atividade do sistema eferente nas propriedades mecânicas da cóclea de ouvintes normais. [Age impact on the efferent system actitivies in co- chlear mechanical properties in normal hearing individuals]. Brazilian Journal of Otorhinolaryngology, 75(3), 340-344. http://doi.org/10. 1016/S1808-8694(15)30648-0

Organización Mundial de la Salud (1999). Guías para el ruido urbano [Guidelines for urban noise]. Londres, Reino Unido.

Organización Mundial de la Salud (2015). $1100 \mathrm{mi}$ llones de personas corren el riesgo de sufrir pérdida de audición [1.1 billion people are at risk of hearing loss]. Recuperado el 22 de junio de 2016 de http://www.who.int/mediacentre/ news/releases/2015/ear-care/es/

Portnuff, C.D.F., Fligor, B.J. \& Arehart, K.H. (2011). Teenage use of portable listening devices: A hazard to hearing? Journal of the American Academy of Audiology, 22, 663-677. http:// doi.org/10.3766/jaaa.22.10.5

Rajan, R. (2000). Centrifugal pathways protect hearing sensitivity at the cochlea in noisy environments that exacerbate the damage induced by loud sound. The Journal of Neuroscience, 20, 6684-6693.

Rasmussen, G.L. (1946). The olivary peduncle and other fiber projections of the superior olivary complex. Journal of Comparative Neurology, 84, 141220. http://doi.org/10.1002/cne.90084 0204

Sadhra S., Jackson, C.A., Ryder, T. \& Brown ,M.J. (2002). Noise exposure and hearing loss among student employees working in university entertainment venues. The Annals of Occupational Hygiene, 46, 455-463. http://doi.org/10. 10 93/annhyg/46.5.455

Schuschke, G., Rudloff, F., Grasse, S. \& Tanis, E. (1994). Unterschungen zu Ausmass und mglichen Folgen jugendlichen Musikkonsums Teil I [Studies on the extent and possible consequences of adolescent music consumption]. Zeitschrift fur Lämbekämpfung, 41, 121-128.

Scientific Committee on Emerging and Newly Identified Health Risks (2008). Potential health 
risks of exposure to noise from personal music players and mobile phones including a music playing function: Preliminary report. Recuperado el 17 de noviembre de 2010 de http://ec. europaeu/health/ph_risk/committees/04_sce nihr/docs/scenihr_o_017.pdf

Serra, M.R., Biassoni, E.C., Carignani, J.A., Minoldo, G., Franco, G., Serra, S., Pollet, A., Joekes, S. \& Blanch, N. (1998). Propuesta metodológica para el estudio de los efectos auditivos de la música a altos niveles sonoros en adolescentes [Methodological proposal for the study of auditory effects of music at high sound levels in adolescents]. Fonoaudiológica, 44(3), 52-60.

Serra, M.R., Biassoni, E.C., Hinalaf, M., Abraham, M., Pavlik, M., Pérez Villalobo, J., Curet, C., Joekes, S.,Yacci, M.R. \& Righetti, A. (2014). Hearing and loud music exposure in 1415 years old adolescents. Noise \& Health, 16, 320-330. http://doi.org/10.4103/1463-1741. 140512

Serra, M.R., Biassoni, E.C., Hinalaf, M., Pavlik, M., Villalobo, J.P., Curet, C., Minoldo, G., Abraham, S., Moreno Barral, J., Reynoso, R., Barteik, M. E., Joekes, S. \& Yacci, M.R. (2007). Program for the conservation and promotion of hearing among adolescents. American Journal of Audiology, 16, 158-164. http://doi.org/10.10 44/ 1059-0889(2007/020)

Serra, M.R., Biassoni, E., Ortiz Skarp, A., Serra, M. \& Joekes, S. (2007). Sound immission during leisure activities and auditory behaviour. Applied Acoustics, 68(4), 403-420. http://doi. org/10.1016/j.a pac oust.2006.03.002

Serra, M.R., Biassoni, E.C., Ritcher, U., Minoldo, G., Franco, G., Abraham, S., Carignani, J.A., Joekes, S. \& Yacci, M.R. (2005). Recreational noise exposure and its effects on the hearing of adolescents. Part I: An interdisciplinary longterm study. International Journal of Audiology, 44(2), 65-73.
Sliwinska-Kowalska, M. \& Kotylo, P. (2002). Occupational exposure to noise decreases otoacoustic emission efferent suppression. International Journal of Audiology, 41, 113-119.

Taranda, J., Maison, S.F., Ballestero, J.A., Katz, E., Savino, J., Vetter, D.E., Boulter, J., Liberman, M.C., Fuchs, P.A. \& Elgoyhen, A.B. (2009). A point mutation in the hair cell nicotinic cholinergic receptor prolongs cochlear inhibition and enhances noise protection. PLOS Biology, 7(1), 71-83. http://doi.org/10.1371/ journal.pb io. 1000018 .

Valeiras, M., Dios, C., Porto, I. \& Labella, T. (2005). Estudio del sistema olivococlear medial mediante la supresión contralateral de las otoemisiones acústicas [Study of medial olivococlear system trough contralateral supression]. Revista Internacional de Otorrinolaringología, 32(3), 122-129.

Vogel, I., Brug, J., van der Ploeg, C. \& Raat, H. (2011). Adolescents risky MP3-player listening and its psychosocial correlates. Health Education Research, 26(2), 254-264. http://doi.org/10. 1093/her/cyq091

Vogel, I., Verschuure, H., van der Ploeg, C., Brug, J. \& Raat, H. (2009). Adolescents and MP3 Players: Too many risks, too few precautions. Pediatrics, 123(6), 953-958. http://doi.org/10. 1542/peds.2008-3179.

Vogel, I., Brug, J., Hosli, E., van der Ploeg, C. \& Raat, H. (2008). MP3 players and hearing loss: Adolescents' perceptions of loud music and hearing conservation. The Journal of Pediatric, 156(3), 400-404. http://doi.org/10.1016/j.jpeds. 2007.07.009.

Wagner, W., Heppelmann, G., Kuehn, M., Tisch, M., Vonthein, R. \& Zenner, H.P. (2005). Olivocochlear activity and temporary threshold shiftsusceptibility in humans. Laryngoscope, 115 (11), 2021-2028. http://doi.org/10.1097/01.ML G.0000181463.16591.A7 
Wallhagen, M.I., Strawbridge, W.J., Cohen, R.D. \& Kaplan, G.A. (1997). An increasing prevalence of hearing impairment and associated risk factors over three decades of the Alameda County Study. American Journal of Public Health, 87, 440-442.

Warr, W.B. \& Guinan, J.J. (1979). Efferent innervation of the organ of Corti, two separate systems. Brain Research, 173(1), 152-155.

Werner, A.F. (2001a). Los mecanismos protectores de la cóclea ante el ruido [Protective mecanism of the cochlea for noise]. Fonoaudiológica, 47 (3), 42-49.

Werner, A.F. (2001b). Teoría y práctica de las otoemisiones acústicas [Theory and practice of otoacustic emissions]. Buenos Aires, Argentina: Edición del Autor.

Werner, A.F. (2006). Teoría y práctica de las otoemisiones acústicas [Theory and practice of otoacustic emissions]. Buenos Aires, Argentina: Edimed: Ediciones Médicas.
West, P.D. \& Evans, E.F. (1990). Early detection of hearing damage in young listeners resulting from exposure to amplified music. British Journal of Audiology, 24, 89-103. http://doi.org/1 $0.3109 / 03005369009077849$

Widen, S. (2006). Noise and music - A matter on risk perception? Unpublished $\mathrm{PhD}$ dissertation. Department of Psychology. University of Göteborgs, Sweden.

Widen, S. \& Erlandsson, S. (2007). Risk perception in musical setting - A qualitative study. The International Journal of Qualitative Studies on Heath and Well-being, 2(1), 33-44. http://doi. org/10.1080/17482620601121169

Zenker, F., Altahona, M.P. \& Barajas, J.J. (2001). La exposición a ruido por actividades de ocio en adolescentes [Exposure to noise in recreational activities]. Revista de Logopedia, Foniatría y Audiología, 21, 173-180.

Centro de Investigación y Transferencia en Acústica (CINTRA) Universidad Tecnológica Nacional (UTN) Facultad Regional Córdoba Córdoba República Argentina

Fecha de recepción: 12 de mayo de 2016 Fecha de aceptación: 19 de septiembre de 2016 